E. Fest, K. Shea, B. Domer and I. F. C. Smith. Adjustable tensegrity structures, J of Structural Engineering, vol. 129, num. 4, p. 515-526, 2003. http://cedb.asce.org Copyright ASCE

\title{
Adjustable tensegrity structures
}

\author{
Etienne Fest ${ }^{1}$, Kristi. Shea ${ }^{2}$, Bernd Domer ${ }^{3}$ and Ian F.C. Smith ${ }^{4}$
}

Keywords: tensegrity, active structure, full-scale testing, non-linearity

\begin{abstract}
A tensegrity is a lightweight space structure consisting of compression members surrounded by a network of tension members. They can be easily dismantled and therefore provide innovative possibilities for reusable and modular structures. Tensegrities can adapt their shape by changing their self stress, and when equipped with sensors and actuators, they can adapt to changing environments. A full-scale prototype of an adjustable tensegrity has been built and tested at Swiss Federal Institute of Technology (EPFL). This paper begins with a description of important aspects of the design, assembly and static testing. Tests show that the structure behaves linearly when subjected to vertical loads applied to a single joint. Non-linearities are detected for small displacements - for loads applied to several joints and for adjusting combinations of telescoping compression members. To predict behavior, dynamic relaxation - a non-linear method - has been found to be reliable. Appropriate strut adjustments found by a stochastic search algorithm are identified for the control goal of constant roof slope and for the load conditions studied. When adjusting struts, an excessive number of adjustable members does not necessarily lead to improved performance.
\end{abstract}

\section{Introduction}

Tensegrity structures are intriguing for their unique visual qualities and topological characteristics (Motro 1997). The term tensegrity is a concatenation of tensile-integrity as termed by one of the inventors, R. Buckminster Fuller (1962), who described them as "small islands of compression in a sea of tension". This is a description of the visual impact of a network of light tension members that provide rigidity to a limited number of discontinuous compression members. Some researchers have found that discontinuous compression is not a necessity for creating a tensegrity and that more efficient structures can be built if compression elements are allowed to join (Robbin 1996). However, defining a tensegrity structure as any self-stressing structure composed of struts and cables would include structures such as a bicycle wheel since spokes could be cables and struts could be rim segments. In order to add precision in terminology, a definition has been proposed by Motro (2001) who describes a tensegrity as a system, in a stable, self-equilibrated state that contains a discontinuous set of components in compression inside a network of components in tension.

Several independent efforts have contributed to the invention and development of tensegrity structures. Le Ricolais, who designed many unusual lightweight cable-strut structures from 1928 (Picon 1997), is believed to have created precursors to tensegrity structures. The sculptor Kenneth Snelson started in 1948 with sculptures employing discontinuous compression (Snelson 1965) and has continued to construct innovative structures (Snelson 1998). Emmerich (1964) applied for a patent for a similar type of structures in France, almost at the same time as Fuller applied in the USA. Applications of the tensegrity principle in nature have recently been found through studies that use a tensegrity model to describe observations of how cells respond to stress (Ingber 1998).

Whether the definition includes continuous or discontinuous compression, tensegrities exhibit unique behavioral properties. An advantage of this class of lightweight structure is characteristic of self-stressing systems. In contrast to tensile structures, such as tensile membrane structures, tensegrities do not need costly anchorages. Moreover, they can easily be dismantled and therefore, provide innovative possibilities for reusable, modular structures.

Transforming tensegrities from sculptures into practical structural alternatives is a challenge that began with several studies of their geometric, non-linear behavior and states of self-stress. As tensegrities are inherently unstable, Calladine (1977) showed that one self-stress state could prevent several mechanisms. Pellegrino and Calladine then developed a matrix method for the determination of the number of self-stress states and mechanisms for general

\footnotetext{
${ }^{1}$ Res. Asst., Structural Engineering Institute, IMAC-IS-ENAC, 1015 Lausanne, EPFL, Switzerland., etienne.fest@epfl.ch

${ }^{2}$ Lecturer, Cambridge University Engineering Department, Cambridge, UK, ks273@eng.cam.ac.uk

${ }^{3}$ Res. Asst., Structural Engineering Institute, IMAC-IS-ENAC, 1015 Lausanne, EPFL, Switzerland, bernd.domer@epfl.ch

${ }^{4}$ Prof. M.ASCE, Structural Engineering Institute, IMAC-IS-ENAC, 1015 Lausanne, EPFL, Switzerland, ian.smith@epfl.ch
} 
tensegrities (Pellegrino and Calladine 1985). More recently, Vassart et al (2000) have developed simple tests to distinguish between first order mechanisms and mechanisms of higher order.

Research in the domain of tensegrity structures is quite active. However, it concentrates mainly on theoretical studies of the mechanics involved. Often, only small-scale models are used for tests (Pellegrino 1992, Motro 1992 and Hanaor 1988). Tests on full-scale structures, as presented in this paper, are rare. Reasons for the lack of test data are related to the fabrication assembly process as well as to the lack of rigidity until prestress is added. Crosnier et al. (1997) bring out these issues saying that the root of the problem is the joint; they consider joint design to be the biggest challenge in constructing a full-scale tensegrity. Although it can be said that this is the case for all space structures, tensegrities present particular challenges. Joints need to be pin-jointed, modular and light: thus, taking advantage of tensegrities' ease of dismantling and potential reuse.

In addition to the complexities of constructing the structure itself, simulation of the behavior is not straight-forward. As a subclass of cable structures, tensegrities exhibit geometric non-linear behavior. Furthermore, as common with other pin-jointed structures, Salama et al. (1993) highlighted that nodal friction should be included in the simulation of a truss structure for precision control. Finally, for full-scale construction, assembly sequences, fabrication tolerances and construction lack-of-fit need to be considered.

During their service lives, all structures have to withstand changing environments. In contrast with more rigid structures, tensegrities provide possibilities to adapt by changing their self-stress. While structures could be designed to adapt only to known environments, adaptation may also be possible for conditions that are difficult to quantify, thus preventing possible damage and collapse.

Changing the self-stress to adapt to changing environments requires tensegrities to be equipped with sensors and actuators. Sultan (1999) formulated control techniques that use a minimal energy optimization. Skelton et al. (2001) built on this work and provided a closed mathematical formulation for the simulation of active tensegrity structures. Work has also been carried out to control the vibrations of tensegrities (Djouadi 1998). Here, four tensegrity modules were used to form a cantilever beam that controls vibration response by changing strut lengths. Controlling larger movements in tensegrity structures also provides interesting perspectives in the field of deployable structures (Tibert 2002).

Active control of tensegrity structures has been studied mainly through simulation where most work has focused on vibration response. A study of full-scale adjustable tensegrity structures adds insight on non-idealized behavior. This paper presents results from research that explores the feasibility of an adjustable tensegrity structure through:

design, modeling, assembly and testing a full-scale prototype,

evaluating the influence of non-linearity on predicted and experimental behavior, and

identifying the most appropriate active struts for a prescribed set of load cases.

\section{Tensegrity structure}

\section{Description of structural properties}

The topology of the full-scale prototype of tensegrity structure that was designed and built at the Applied Computing and Mechanics Laboratory (IMAC) at EPFL was inspired by an original design by Passera \& Pedretti (Pedretti 1998), Lugano for the Swiss National Exhibition, see Figs. 1 and 2.

The structure consists of three modules. Each module contains six struts and twenty four cables. In contrast with classical tensegrity structures, a central joint is used to reduce the buckling length of the compression members, thereby lowering the required cross-sectional area of the struts. Telescopic struts are used to modify the geometry and self-stress in the system. Six-millimeter diameter stainless steel cables form a double-layered structure, where lateral cables connect top and bottom layers. Each layer is composed of two cable lengths, one forming three isosceles triangles and the other forming an equilateral triangle in the center, see Fig. 1.

The six struts of each module meet at the central joint. This joint has cone-shaped openings where the ball-bearing end of each strut is placed. Transmission of bending moments is thereby suppressed. Nevertheless, one strut of each module is fixed at the central joint, which is necessary for assembly. The other end of the strut consists of a nut/threaded rod system to enable changes in strut length using a simple wrench (Fig. 3.). Changing the strut lengths results in changing the amount of self-stress in the structure. 
All struts are made from fiberglass reinforced polyester tubes. Compared with steel, this material offers a more efficient trade-off between density and Young's modulus. A minimal diameter of the struts is needed for movement of the threaded rod (Fig. 3.) and also this provides sufficient buckling strength, see Table 2. In order to accommodate different connection situations between cables and between cables and struts, a module-connection joint has been created (Fig. 4. and 5.). Mechanical properties of struts and cables, including Young's Modulus, buckling force, elastic limit and fracture of the cables, have been checked through experimental testing of individual elements.

When three modules are connected to create one tensegrity structure, the total number of joints is thirty three and the number of tension and compression elements is 90 . The height of the structure is $1.10 \mathrm{~m}$ covering a total surface area of $9 \mathrm{~m}^{2}$ and weighing $30 \mathrm{~kg} / \mathrm{m}^{2}$. Six degrees of freedom are fixed at the three supports.

\section{Assembly method}

Assembly of the tensegrity structure includes building individual modules, connection of modules to create the desired configuration and the introduction of required self-stress in the structure. The amount of self-stress is prescribed by the change in length of the telescopic struts. Proceeding from an initial strut length of $1296 \mathrm{~mm}$, extending all struts to $1298 \mathrm{~mm}$ stiffens the assembly sufficiently. This state is called $2 \mathrm{~mm}$ self-stress and introduces a uniform compressive force of $3000 \mathrm{~N}$ into all struts.

Assembling the structure is difficult since no intermediate state of the assembly is in static equilibrium. The assembly procedure proceeds according to two criteria:

the top plane of the central joint is always kept horizontal, and

the strut length remains constant.

\section{Constructing the form}

Each module of the structure is assembled independently. Modules are connected later to create the final structure. The two cable layers of each module are composed first. Cables are linked to form the equilateral triangles and the six isosceles; lateral cables are also added. The precision of the element lengths in laboratory conditions is $\pm 0.5 \mathrm{~mm}$.

The struts are then introduced, beginning with the three struts and the central joint, which form the bottom pyramid. Attaching a weight to the central joint stiffens the pyramid. After that, the three upper struts are mounted. At this stage, the form of the module is still undetermined. Stiffness is added by lengthening the telescopic struts stepwise. The sequence in which the struts are lengthened is important, since an asymmetrical sequence could create an imbalance in the central joint and cause structural failure. Once these compression members have introduced the minimal amount of self-stress required, thus determining the form, the mounting-weight can be taken away from the central joint.

After assembling individual modules, they are then connected together to form the final structural configuration. When lateral cables interfere during module connection, they are detached and re-attached once the modules are placed into their final position. Such intervention does not affect structural stability. Finally, the structure is set onto the supports.

\section{Stiffening the assembly}

After construction, imprecision in individual cable lengths can affect the tension of all cables. By measuring the cable frequency with a laser-based method (Cunha and Caetano 1999), tension in individual cables is checked with that calculated using the dynamic relaxation method (see section below). Cable lengths are then modified to correct the tension. The experimental method used, which has been applied successfully to cable stayed bridges, has been adapted for use with tensegrity structures (Baumann et al. 2000).

Cable lengths are modified incrementally, beginning with highly stressed cables and finishing with those having lower stresses (Fig. 5). This iterative process is repeated until the worst relation between measured and calculated tension is less than $20 \%$. During this process, the slope of the upper cable plane is checked to determine the stability of the system. The cables with the highest tension are the cables that form the isosceles triangles. The lateral cables are then tensioned to approximately $80 \%$ of the tension in the isosceles cables whereas the cables of the inner, equilateral triangle are tensioned to approximately $20 \%$. 


\section{Numerical modeling}

The goal of numerical modeling is twofold: (1) to compare measured physical behavior with analytic calculations and (2) to provide a means for predicting the behavioral effect of adjusting telescopic struts. The dynamic relaxation method has been chosen since it is a widely used technique for the analysis of cable and tensile membrane structures. Using telescopic struts to adjust the shape of the structure to changing conditions, appropriate strut movements need to be determined. Stochastic search techniques will be shown to be capable of finding good adjustment solutions for shape control. These aspects are discussed in the next section.

\section{Static analysis: Dynamic relaxation}

The simulation of tensegrity structures must include the effects of geometrical non-linear behavior. In contrast with traditional finite-element calculations where the non-linear terms of the stiffness matrix are calculated and added to a linear stiffness matrix for each iterative step, as a vector method, dynamic relaxation (Barnes 1977) calculates nonlinear behavior directly. The dynamic equation of a damped system with externally applied loads is used to calculate the static behavior of structures:

$\mathrm{p}(\mathrm{t})=\mathrm{M} \ddot{\mathrm{d}}+\mathrm{C} \dot{\mathrm{d}}+\mathrm{Kd}$

where:

$\begin{array}{ll}\mathrm{p}(\mathrm{t}) & \text { time-dependent vector of externally applied load } \\ \mathrm{M} & \text { 'fictitious' mass matrix } \\ \mathrm{C} & \text { matrix of 'fictitious viscous' damping coefficients } \\ \mathrm{K} & \text { stiffness matrix } \\ \mathrm{d} & \text { vector of displacement }\end{array}$

The motion of structural joints is traced over time until the sum of residual forces, represented by the first two terms on the right hand side of Equation 1, in the joints converges to a near zero value. This indicates that the state of equilibrium of the structure has been reached.

Parameters to be set prior to analysis include the fictitious masses $\mathrm{M}$ and fictitious damping coefficient $\mathrm{C}$ of equation (1), as well as the time step $\Delta$ t. Several concepts of choosing these parameters in order to achieve rapid convergence have been compared (Papadrakakis 1981). One of these concepts, kinetic damping, does not require choosing the viscous damping matrix $\mathrm{C}$ in equation (1). The kinetic energy of the undamped structure is traced. Whenever a kinetic peak is detected by a sudden fall of kinetic energy, the iteration steps back to the moment in time where the kinetic peak is assumed to have occurred. At this point, nodal velocities are reset to ' 0 ' and the current coordinates are taken as starting values for the next cycle of iterations (Rossier 1993)

Unilateral rigidity can be introduced with each time step, since no pre-assembled stiffness matrix is used. Whenever a cable receives a compressive force its inner force is set to 0 for the following iterative steps. The dynamic relaxation method provides nodal displacements as well as element forces. A separate form-finding process is therefore not necessary.

\section{Shape adjustment}

The tensegrity structure is equipped with sensors and actuators such that the shape and thus behavior of the structure can be adjusted. The way that shape adjustment is utilized is schematically summarized in Fig. 6. The initial state of the structure is disturbed by a load. The structural response is then introduced as an input in the search box that combines a predictive model and the search algorithm. The search is carried out considering objectives and constraints. The predictive model is analyzed using dynamic relaxation. The search result consists of a set of strut adjustments that is applied to loaded structure. The new structural response is then measured to quantify the effectiveness of the shape adjustment.

Finding a good set of adjustment commands for such a coupled, non-linear system is a complex problem. Analyzing all theoretically possible adjustments leads to an exponentially increasing number of analyses with the number of adjustable struts (Fig. 7). A search is required to determine which strut to adjust and by how much. While the first quantity is naturally discrete, the second is discredited into increments of $0.25 \mathrm{~mm}$ for practical reasons. For the adjustable tensegrity structure described, the search space is discrete and is defined by the number of adjustable struts, 
the range of movement of individual struts and the feasible increment of movement. The number of possible adjustment combinations, $\mathrm{N}$, is then:

$$
\mathrm{N}=\mathrm{P}^{\mathrm{n}}
$$

where $\mathrm{P}$ is the number of possible strut positions, and $n$ is the number of adjustable struts. For this tensegrity structure there are eighteen adjustable struts and the number of possible strut positions is determined by the range of movement, $6 \mathrm{~mm}$, and the smallest practicable movement is $0.25 \mathrm{~mm}$. This results in over $5 \times 10^{20}$ possible combinations. Placing constraints on the number of adjustable struts creates smaller solution spaces.

Searching large solution spaces through stochastic search methods has the potential to identify beneficial adjustment commands (Shea and Smith 1998). For this structure, simulated annealing (Kirkpatrick 1983) will be used. It stems from an analogy to the annealing of metals where temperature schedules are used to control the arrangement of atoms during their crystallization process. It is a step-wise technique that allows consideration of inferior solutions and therefore is able to overcome local minima.

The process is driven by the function:

$$
\mathrm{P}_{\text {accept }}=\mathrm{e}^{-\frac{\Delta \mathrm{C}}{\mathrm{T}}}
$$

The change in the objective function, or cost, between two moves is denoted by $\Delta \mathrm{C}$. $\mathrm{P}_{\text {accept }}$ is compared to a randomly generated value between 0 and 1 and the inferior candidate solution is accepted when the random value is less than

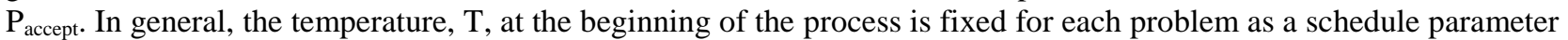
and is then reduced to zero during the optimization process according to an "annealing schedule". During the last section of this schedule, the "freezing" stage, only better moves are accepted and simulated annealing behaves as a descent strategy.

Simulated annealing algorithms are reasonably robust if the parameters controlling the cooling curve are assigned values that reflect the complexity of the solution space. This implementation uses the modified Lam-Delosme annealing schedule to define schedule characteristics (Swartz and Sechen 1990) and the concept of Hustin (1988) move sets to create dynamic rule selection. Generally, larger adjustments are tried in the beginning of the process whereas smaller moves are used towards the end as the solution converges. Further details on the simulated annealing schedule and parameter definition are given in Hustin (1988) and Swartz and Sechen (1990).

\section{Test set up}

\section{Static tests}

The tensegrity structure built at IMAC has been used to perform two different types of tests:

static load tests, and

adjustment of telescopic struts to maintain the initial slope of the structure

In both cases, vertical loads were applied only on joints connecting at least one strut. This is a realistic constraint since in service, no load would be applied to joints that do not have at least one member that is capable of compressive resistance.

The following four load cases are applied (Table 1 and 3).

Joints are loaded symmetrically to simulate a uniform load applied to a roof. Joints 6, 52 and 62 form the largest equilateral triangle of the upper plane (Fig. 8). Joints 5, 48 and 61 (Fig. 8) form a smaller triangle of the upper plane. Both joint sets could be used for attaching a covering material to the structure. The maximum magnitude of the applied load is $860 \mathrm{~N}$. Loads are applied incrementally and simultaneously on each joint in steps of $152 \mathrm{~N}$ and $236 \mathrm{~N}$.

A single joint is loaded to evaluate the influence of an asymmetric load. The maximum magnitude of the load is $1923 \mathrm{~N}$. It is applied incrementally on the joint in steps of $152 \mathrm{~N}$ and $236 \mathrm{~N}$.

The central joint of each module is loaded simultaneously with maximum magnitude of $860 \mathrm{~N}$. It is applied incrementally and simultaneously on each joint in steps of $152 \mathrm{~N}$ and $236 \mathrm{~N}$. Here the load is applied at the connection of only compression members. 
The structure is loaded by adjusting strut lengths. The maximum number of struts that can be adjusted is 15 since it was decided not to adjust the three struts that are connected to measured joints. The maximum movements from the initial position are $+/-3 \mathrm{~mm}$ - with increments of $0.25 \mathrm{~mm}$.

Except for the case of adjusting struts, joints are loaded by a weight that is attached to a cable which is screwed into a thread at the joint center. By adding steel plates to the initial weight, the load can be changed incrementally. For each case, loads are applied three times in order to assess repeatability.

Nine linear variable differential transformers (LVDTs) measured vertical displacements at nine joints, as indicated in Fig. 9 Their nominal accuracy is +/- 10 microns and they are used with a low pass filter to minimize noise problems. An automated data acquisition system has been employed to record the data. Locations of the measured joints have been chosen to investigate the displacements of the three structural levels (Fig. 9). The response joints are symmetrically placed and form the larger equilateral triangle of each level. Movements of joints 3, 50 and 59 indicate lower-level deflections. The joints 7, 54 and 63 are the central joints of each module and their movements show the deflection of the middle level. Movements of joints 6, 52 and 62 illustrate the deflections of the upper level (Fig. 9).

Measured displacements are compared with predictions obtained from the dynamic relaxation method. Input parameters in the analysis model are joint coordinates, connectivity of the elements, material characteristics, boundary conditions of joints, level of pre-stress and applied loads. Three symmetric lower joints are connected to concrete blocks to simply support the structure. Joint 1 is fixed in all directions (X, Y and Z), Joint 51 in the $\mathrm{Y}$ and $\mathrm{Z}$ directions and Joint 60 in Z-direction. The $\mathrm{Z}$-axis is in the vertical direction, the $\mathrm{X}$ and $\mathrm{Y}$ axes are in the horizontal plane of the structure (Fig. 8).

The structure was initially prestressed by lengthening the struts by $2 \mathrm{~mm}$ for the static tests. Struts are lengthened by 2 $\mathrm{mm}$, thus resulting in an initial length of $1298 \mathrm{~mm}$. This level of self-stress represents a compression force of about $7500 \mathrm{~N}$ in struts, and a tension force of about $2200 \mathrm{~N}$ in lateral cables, $650 \mathrm{~N}$ in short cables of the equilateral triangle and $2700 \mathrm{~N}$ in the other cables of the isosceles triangles.

\section{Shape adjustment}

The goal of shape adjustment in this study is to maintain the initial slope of joints of the structure on the upper level where a covering material could be attached. Such criterion is comparable to ponding avoidance measures if the structure is employed as a roof. Joints 5, 48 and 61 are chosen to represent the upper-level slope forming an equilateral triangle as indicated in Fig. 8. They are instrumented with 3 linear variable differential transformers (LVDTs) to measure vertical displacements $\delta_{\mathrm{i}}$. The shape adjustment task consists of adjusting the lengths of a number of telescopic struts such that the initial slope is maintained (Fig. 10). Deviation from the initial slope, called the response error, is calculated through a root mean square of the relative vertical displacements of the three joints:

$$
\text { response error }=\sqrt{\frac{\left(\delta_{5}-\delta_{48}\right)^{2}+\left(\delta_{5}-\delta_{61}\right)^{2}+\left(\delta_{48}-\delta_{61}\right)^{2}}{3}}
$$

For the tensegrity configuration presented, eighteen telescoping struts have potential to be adjusted. Considering that adjustment is not performed on three struts that are fixed to measured joints, the number of adjustable struts reduces to 15. The telescoping struts are initially set at their nominal length of $1296 \mathrm{~mm}$. For these tests a $4 \mathrm{~mm}$ pre-stress position, which corresponds to struts lengths of $1300 \mathrm{~mm}$, is considered as the zero position, $P_{o}$ (Fig.3.). Each strut can then be either lengthened or shortened in discrete increments to change the system self-stress.

In this study, adjustments are made manually. For initial testing, the movement increment is determined from the lead screws and the possible accuracy level by manually lengthening and shortening the struts. One full turn of the screw lengthens the strut by $1.5 \mathrm{~mm}$. A hexagonal bolt allows reliably repeatable turns of one bolt side, setting the accuracy of the movement to $0.25 \mathrm{~mm}$. Considering maximum stress and critical buckling force, changes in strut length are restricted to $+/-3 \mathrm{~mm}$. The allowed stress in cables is $\sigma_{\text {allow-cable }}=613 \mathrm{~N} / \mathrm{mm}^{2}$; this value incorporate a safety factor of two against yielding. The allowed buckling force in struts is $f_{\text {allowed }}=20000 \mathrm{~N}$, which is based on experimental tests on $1 \mathrm{~m}$ struts (Marguccio et al 2000). The allowed buckling force is reached before the maximum allowable fracture force, $f_{\text {fracture }}=43000 \mathrm{~N}$.

When determining adjustment solutions using simulated annealing, two numerical models are used to predict the change in behavior of the structure due to changing the lengths of a maximum number of struts. Response displacements $\delta_{\mathrm{i}}$ can be predicted from numeric structural analysis as well as by measuring the structure under 
loading. The analytic model is based on the geometry, topology and material properties of the structure analyzed using the dynamic relaxation method. The calibrated model extends the analytic model to include a correction based on measurements obtained from the structure. A stochastic search method, simulated annealing, (described previously) is able to employ either predictive model for determination of the strut adjustments. The objective is to minimize the response error defined in Equation 4. More details on computational aspects of shape adjustment are given in Shea et al. (2002).

Given a set of adjustments from the search, the experimental testing procedure is described in Fig. 11. For testing a single set of hypothetical adjustments for struts A to Z, the procedure is divided into three steps:

Step 1: The initial state of the structure is measured. The heights of the three response joints 5,48 and 61 are measured by LVDTs

Step 2: The structure is loaded and the displaced state is measured. The structure is unloaded and loaded again to examine whether behavior is repeatable.

Step 3: Displacements are measured and the search is invoked. The search provides a set of commands for extending and contracting the active struts. Since the struts are adjusted manually, the commands can not be applied simultaneously, and thus a sequence is chosen for adjusting the strut lengths. The default sequence starts with strut index A and changes are applied in increasing alphabetic order until all changes are made. A numerical analysis is made to predict the behavior after each change is made to ensure that no member will fail during the sequence. If a strut is near the buckling limit or a cable near the stress limit at any point in the sequence, a different sequence is adopted.

After a safe sequence is chosen, each strut is adjusted and the displacements of the response joints are measured. Again, adjustments in the sequence can be made if any change may cause failure in any part of the structure. After all changes are made, the final state is measured and compared to the prediction. To return to the initial state, the steps are applied in exact reverse order. The final unloaded state is then measured and is compared to the initial state measured.

Four load cases are considered, including both top/center and asymmetric loads (Table 1.). Two cases of control variables are studied, the first allowing all 15 struts to be active while the second limits the number of active struts to two symmetric struts per module. The second case has been formulated to guide decisions related to which struts will be motorized in a later study of active structural control. The choice of the active struts is constrained by symmetry, since a similar arrangement should be applicable when enlarging the structure to more than 3 modules.

\section{Results}

The behavior of the adjustable tensegrity built is in general non-linear and complex. This section describes an investigation of the behavior of the structure through a comparison of experimental testing and numerical analysis. Results are presented in increasing complexity of the load case. The first and second load cases are vertical loads applied to single joint locations. The third load case is simultaneous vertical loads at different joint locations. To investigate the structural response to intentional adjustments, the fourth load case consists of multiple changes in strut lengths, which results in loads applied simultaneously at multiple joints. Experimental tests were carried out three times for each load case. In the figures, continuous lines are used to show the displacements calculated by the analytic model and data points represent the displacements measured.

\section{Correlating the numerical model with test results}

The first load case is a single vertical load applied to the central joint of module 2, Joint 7. Descriptive displacements of the structure for the load case are illustrated in Fig. 12. Fig 12a illustrates the varied response of the three levels of the structure and the three modules. Fig. 12b shows the displacements of the loaded joint (Joint 7). Fig. 12c shows, of all joints, the maximum displacement occurs at Joint 3, which belongs to a different module, module 3 , than the loaded joint. It is located on the bottom level and is the farthest joint from the loaded joint as well as from the supports. The maximum displacement in the structure is in the same direction as the loading direction. Fig. 12d shows the displacement of a joint on the upper level of the structure (Joint 52). Even though the joint measured belongs to the same module as the loaded joint, the displacements are in the opposite direction. Fig. 12e shows the displacements of another central joint (Joint 63) located in module 1. The displacements here are much smaller than the loaded joint itself and are in the opposite direction. 
Results demonstrate that the displacement evolution is continuous with increased loading. The non-linearity is continuous, but small and only becomes meaningful for loads above $981 \mathrm{~N}$. Even including non-linearity, measurements agree well with the analytic model. Agreement decreases by less than 95\% after $981 \mathrm{~N}$ due to nonlinearity but is not lower than $90 \%$.

All figures demonstrate that the experimental technique is reliable as measurements of the three independent tests are comparable. Fig. 12c shows about 15\% difference between the series of data points for loads $1802 \mathrm{~N}$ and $1681 \mathrm{~N}$, illustrating that repeatability is the best for loads below $981 \mathrm{~N}$. Relaxation in cables can also influence repeatability of the behavior.

\section{Investigating local linearity}

The second load case is a single asymmetrical load applied at Joint 62, a top edge joint. Displacements that illustrate the local behavior of the three levels and modules of the structure for this load case are shown in Fig. 13. Fig 13b shows the displacements of the loaded joint (Joint 62). Fig. 13c shows, of all measured joints, the maximum displacement occurs at Joint 59, which belongs to the lower level of the loaded module. The maximum displacement is in the same direction as the loading direction. This load case yields larger maximum displacements than the previous load case (compare Fig. 13c with Fig. 12c.). Fig. 13d shows the displacement of an asymmetric joint (Joint 52) in module 2, at the upper level of the structure where the displacements are smaller and in opposite direction than the loaded joint. Fig. 13b shows the displacements of a joint on the middle level of the structure (Joint 7) which are in the same direction of the loaded joint.

Again, all figures demonstrate a good repeatability of measurements where the difference between the series of data points is less than $5 \%$. Measurement results also demonstrate an excellent agreement with the analytic model where the difference between experimental data and the analysis is less than 5\%. Displacements increase linearly as the load increases to $860 \mathrm{~N}$. Comparing all figures, the linearity is not influenced by a coupled system given that measurement locations are on different modules. A similar linearity is illustrated for the previous load case regarding the same load range. Comparing single joint load cases, an asymmetrical load not applied at central joint causes larger displacements.

The third load case is simultaneous symmetric loading of three edge joints, which form the largest triangle of the upper level of the structure. Each joint belongs to a different module; Joint 62 belongs to module 1, Joint 52 belongs to module 2, and Joint 6 belongs to module 3.

Descriptive displacements of the three levels of the structure and the three modules for this load case are illustrated in Fig. 14. Figs. 14b and 14c illustrate the displacements of two loaded joints (Joint 6 and 62). They show a similar behavior of both joints given a symmetric load case. Similar behavior is seen at Joint 52 since the response triangle translates down under load and remains horizontal. Loading symmetrical joints, the structure equilibrates, and displacements are smaller than loading a single joint. For the same load magnitude, the displacement of a loaded joint for the symmetric load case (Fig. 14b) is almost 5 times smaller than the displacement measured for an asymmetric load case (Fig. 13b). Fig. 14d shows the displacement of a joint in the middle level of the structure (Joint 54) which is smaller than upper level displacements. The displacement of the lower level (Fig. 14e) is the smallest.

All figures show that the correlation between the displacements obtained using the analytic model and measurements is not as reliable as in the previous tests. Fig. 14d shows approximately a $25 \%$ difference between measurement data and analytical predictions. This is likely due to the small displacements involved. Several loaded joints also increase the influence of joint friction and cable relaxation when compared with single joint loads. For the load range (0-860N) in all figures, displacements increase linearly with an increase in load. This linearity does not depend on the level of structure and the module measured.

To summarize the results of the first three load cases, linear behavior may be assumed in the load range of $0 \mathrm{~N}$ to $860 \mathrm{~N}$ for single joint loads and symmetric multiple loads. Also, the analytic model, which uses dynamic relaxation, correctly predicts the behavior of the structure. Repeatability could be influenced by joint friction and cable relaxation. Flexibility is most prominent when the structure is loaded asymmetrically.

\section{Investigating non-linear behavior}

Superposition requires linear behavior. Although local linearity has been observed for certain load configurations, the generality of such behavior requires investigation before this assumption can justify avoidance of lengthy iterative 
analyses. The assumption of superposition, through adding displacements caused by vertical loads (or strut length changes) is evaluated in this section.

\section{Elastic superposition of single joint loads}

Assuming the linear behavior that was described in the previous section, this section describes an investigation of the use of elastic superposition to predict structural behavior when several struts are adjusted. A load case of three symmetric joints loaded simultaneously is compared with the elastic superposition of results found by tests that load the same three joints individually.

Fig. 15 shows the displacements of joints 59 and 3 for the structure loaded symmetrically on the joints 5, 48 and 61 . The three loaded joints form an equilateral triangle on the upper level of the structure, which is smaller and further away from the supports than the previous load case. The structure is more sensitive to this load case than the previous (Fig. 14a et Fig. 15a). In both figures 15b and c, the solid circles show the displacements found from elastic superposition of independent, single joint loads. Given three independent loads on joints 5, 48 and 61, the displacements measured for each load case are added to simulate the total symmetric load.

Both figures 15b and c show that elastic superposition does not predict the real behavior. Using elastic superposition, the displacements of the structure are smaller than measured. While individual single asymmetric loads may result in larger displacements than the total symmetric load, as shown in the previous section, these can occur in opposite directions making their combination smaller. These results agree with the widely-known fact that tensegrity structures exhibit geometrically non-linear behavior. So for single vertical loads, even when behavior is linear, a new analysis would have to be carried out for each new load case.

\section{Strut adjustment}

Load cases that involve vertical loads have been explored in the examples provided thus far. Applying a set of changes in lengths on struts is a way of loading the structure that is of particular interest for shape adjustment. Given linear behavior under single vertical joint loads, a plausible assumption can be made that for small changes of strut lengths linear superposition of associated displacements will yield a good approximation of their combined effect. To achieve this, each joint displacement of the structure is calculated using the analytic model for a $1 \mathrm{~mm}$ change in the length of a single strut to create an array of resulting displacements. Performed for a $1 \mathrm{~mm}$ change in length of all struts individually, and considering three dimensions, the size of the matrix is the number of struts times by three the number of response joints, which in this case is [18×99]. Dividing each member of the influence matrix by the change in strut length produces a non-dimensional matrix that can be used for calculating displacements of joints due to changing combinations of strut lengths. This matrix enables a faster simulation of the behavior of the structure than performing a full analysis. Since the most beneficial strut adjustments are determined by stochastic search, which generally involves lengthy evaluations of several ten s of thousands of possibilities, more rapid calculation methods are attractive.

Fig. 16 illustrates an example of the behavior of the tensegrity structure as determined by the structural response defined in Equation 4 (Shea et al. 2002.). The dotted line displays the structural response predicted using the influence matrix previously described and the solid line is the result of the analytic model, which employs dynamic relaxation after each adjustment. The figure shows that moving struts incrementally, results in a non-linear evolution of the structural response. The magnitude of the changes in length of individual struts is within a range of $+/-3 \mathrm{~mm}$. Moving fifteen struts creates large displacements sufficient to improve the response by $10 \mathrm{~mm}$. The correlation between analytic and experimental models is reliable. It is noteworthy that as each new strut is adjusted, the difference between analytic and experimental models does not always increase. After fifteen changes, the difference between measurements and predictions is less than $10 \%$.

Calculating the deflection of the structure for the particular load case with a linear influence matrix overestimates the response of the structure to strut adjustments. The variation in response with the experimental results is too important to be an acceptable model for predicting structural behavior. Even for small strut movements a linear assumption of behavior is not accurate.

In summary, the tests which studied non-linear behavior in the adjustable tensegrity found the following:

an analytic model calculated by the dynamic relaxation method is reliable to make predictions of the behavior of the tensegrity structure; 
simplifications involving assumptions of linear behavior for small movements are not accurate enough to predict the behavior in general conditions of vertical loading and for strut adjustments;

the experimental technique used was found to be sufficiently robust and repeatable.

These observations provide important information to move towards adjusting the shape of the tensegrity structure intentionally to achieve geometric performance goals.

\section{Shape adjustment}

The goal of shape adjustment can be defined as maintaining the slope of the upper level of the structure through changing combinations of strut lengths. Two possibilities for adjustment are considered: the first allowing five movable struts per module (Fig. 17) and the second allowing only two symmetric movable struts per module (Fig. 18). The response of the adjusted structure, as calculated by Equation 5, is used to evaluate the effectiveness of a set of adjustments.

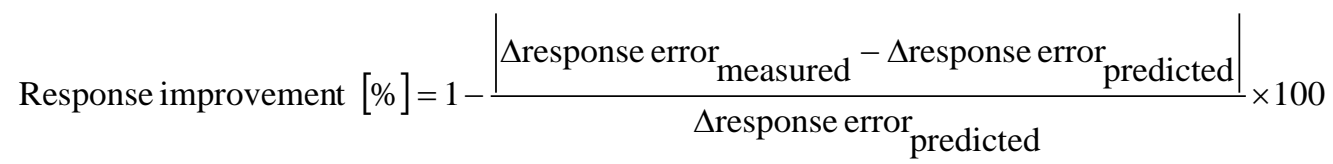

where $\Delta$ response error $=\mid$ response error $_{\text {initial }}-$ response error $_{\text {final }} \mid[\mathrm{mm}]$

The measured response error (Equation 4) is compared with predictions by two models. This comparison is evaluated in a ratio (Equation 5) called the response improvement. Values of response improvement close to $100 \%$ indicate good predictions of behavior of the upper level of the structure. The dark column represents the analytic model and the white column the calibrated model. Shape adjustment is tested for four load cases. It is noteworthy that all adjustment sets required the same search time (execution time of the simulated annealing algorithm) since the search space is the same size. For all load cases, adjusting struts resulted in changes in the upper level slope toward the performance goal of restoring the initial slope. It shows that the combination of simulated annealing with dynamic relaxation has potential for identifying good sets of adjustments even in situations of non-linear behavior and exponential search spaces. The main influencing factors on the success of predicted improvements that will be discussed are the following: (1) the amount of initial response error that needs to be adjusted for, (2) the position of the applied load(s), and (3) the relation between the analytic and calibrated model for the load case involved.

Fig. 17 demonstrates that for both models, the set of adjustments is the most accurate (95\%) for load case 1, then for load case 2 (78\%), then load case 3 (58\%) and finally, for load case 4 (46\%). This shows that in practice, shape adjustment using 5 telescopic struts per module is most appropriate for asymmetric loads. This could mean that shape adjustment is more effective when displacements are large.

For load case 1, both prediction models lead to a near complete agreement with measurements of improvement (95\% and $96 \%$ ). For load case 3 and 4, measurements revealed only $16 \%$ and $35 \%$ of the analytical predicted improvement, while use of the calibrated model resulted in $58 \%$ and $46 \%$ response improvement. Although making predictions with the calibrated model is somewhat effective, making predictions with the analytic model is not reliable for a top/center load case, (Load case 3 and 4). In situations where achieving the performance goal with just one adjustment set is not a success, opportunities exist for making an iterative adjustment beginning with the new deformed shape. Iterative adjustment may lead to an active structure that can be modified step by step provided that convergence is certain.

For the case of two adjustable struts per module (Fig. 18) and considering both prediction models, the set of adjustments is the most effective for the load case $4(100 \%)$, then load case $1(99 \%)$, then load case $2(97 \%)$, and finally load case 3 (81\%). This statement shows that shape adjustment using 2 telescopic struts per module is effective for both asymmetric and top/center load cases.

The effectiveness ranking given by the above paragraph was based on results of predictions using the calibrated model (Fig. 18). Using analytic model enables a good (78\% and $81 \%$ ) shape adjustment for the asymmetric load case, but it is not accurate enough for the top/center load case (Load case 3 and 4), (54\% and 30\%). Considering two adjusted struts per module and using a model calibrated with measurements provides the greatest accuracy. Once again, for cases where adjustments do not meet performance goals, continuing to adjust the structure iteratively could lead to better response improvement. Since a calibrated model allows better adjustments, further response improvement may 
be possible using more sophisticated ways to correct predictions, such as artificial neural networks. This is the subject of current research. Regarding Figs. 17 and 18, both two adjustable members and five adjustable members are suitable for the asymmetric load case (Load case 1). Comparing Figs. 17 and 18 limiting the number struts to adjust from five to two results in better improvements in response, particularly for the calibrated model. This non-intuitive observation can be explained through a common observation in control engineering. Use of an over-conditioned model may introduce larger global error through cumulating errors on a high number of active elements. Further description of the computational control framework can be found in Shea et al. (2002).

The second possibility of shape adjustment, limiting the number of active struts to two for each module, was motivated by the selection of struts that will have actuators. For the results shown in Fig. 18, shape adjustment for load cases one through three required adjustments to struts B3 and B4 and their symmetric counterparts in other modules. Shape adjustment for load case four required adjustments to struts B3 and B6 and their symmetric counterparts (see Fig. 8. for strut indices). Further tests are underway to limit the number of active struts to only two symmetric per module across all load cases. For example, a similar quality solution was found for load case 4 when the generation of solutions was limited to struts B3 and B4.

\section{Conclusions and discussion}

Active tensegrity structures have the potential to widen the scope for innovative, lightweight and reusable structural systems. Through the construction and testing of an adjustable full-scale prototype, it has been observed that the structure behaves linearly when subjected to vertical loads on single joints. Non-linear behavior occurs for the case of small deflections, loads applied to several joints and adjusting combinations of telescoping struts. Simulation of the behavior using dynamic relaxation proved successful for predicting the response of the prototype under symmetric and asymmetric loading as well for simultaneous strut adjustments. It is unlikely that such a highly coupled, flexible and non-linear structure would be able to satisfy service criteria without adjustment in practical situations, especially under asymmetric loading. It was found that when adjusting struts to meet serviceability criteria, increasing the number of adjustable struts from two to five per module does not necessarily lead to improved performance. This work contributes to the development of structures that, through computational control and recording previous adjustments, will improve their performance during service life.

\section{Acknowledgments}

The authors would like to thank the Swiss National Science Foundation and Maag Technic for their support. We are also grateful to Passera \& Pedretti SA, Lugano, S. Rossier, Y. Perelli, and R. Delez, for their contributions as well as to D. Marguccio and F. Baumann for their assistance related to experimental aspects.

\section{References}

Barnes M.R., (1977). “Formfinding and Analysis of Tension Space Structures by Dynamic Relaxation.” The City University, England.

Baumann F., Fest E., and Smith I. F. C., (2000). "Développement d'un Système de Mesure pour Evaluer la Tension dans les Câbles du Tensegrité.” Travail de Semestre, Swiss Federal Institute of Technology, Switzerland (in French), p. 15.

Calladine C.R., (1978). "Buckminster Fuller's "Tensegrity" Structures and Clerk Maxwell's Rules for the Construction of Stiff Frames.” Int. J. Solid Structures, 14, 12-17.

Casas J.-R., (1994). “A Combined Method fo Measuring Cable forces: The Cable-Stayed Alamillo Bridge.” International Association for Bridge and Structural Engineering, 4, 235-240.

Crosnier B., and Cevaer F., (1997). “Le noeud problème.” Systèmes de Tensegrité, Edition de l'Esperou, Montpellier, France (in French), 31-35.

Cunha A., and Caetano E., (1999). "Dynamic Measurements on Stay Cables of Cable-stayed Bridges Using an Interferometry Laser System.” Experimental Techniques, May/June, 38-43.

Djouadi S., (1998). "Le Contrôle des Structures Tensegrité et les Systèmes tensegrité.” Doctoral thesis, Université Montpellier II, Laboratoire de Mécanique et Génie Civil, Montpellier, France ( in French), p.134. 
Dowsland K., (1995). “Simulated Annealing.” Reeves, C. (ed.): Modern heuristic techniques for combinatorial problems, McGraw-Hill, 20-69.

Emmerich D. G., (1964). “Construction de Réseaux Autotendants.” Brevet N¹,377,290, Brevet délivré par le ministère de l'industrie, France ( in French).

Fuller R. B., (1962). “Tensile Integrity Structures.” U. S. Patent N³,063,521.

Hanaor A., (1988). "Prestressed Pin-Jointed Structures-Flexibility Analysis and Prestress Design.” Computers \& Structures, 28, 757-769.

Hustin S., (1988). " A New Standard Cell Placement Program Based on the Simulated Annealing Algorithm.” Master of Science, University of California, Department of Electrical Engineering and Computer Science, Berkeley, USA.

Ingber D.E., (1998). “The Architecture of Life.” Scientific American”, January, 30-39.

Kirpatrick S., Gelatt C. D., and Vecchi P., (1983). “Optimization by Simulated Annealing.” Science, 20, 671-680.

Marguccio D., Fest E., and Smith I. F. C., (2000). "Etude de la Conséquence de Chocs sur les Barres en Fiberline Montées dans les Structures Tensegrités.” Travail de Semestre, Swiss Federal Institute of Technology, Switzerland ( in French), p.15.

Motro R., (1997). “Tensegrity Systems and Structural Research.”, Current and Emerging Technologies of shell and Spatial Structures, IASS, 161-173.

Motro R., (1992). “Tensegrity Systems: State of the Art.” Int. J. of Space Structures, 7(2), 75-83.

Motro R., and Raducanu V. (2001). “Tensegrity Systems and Tensile Structures.” Extended Abstracts International Symposium on Theory, Design and Realization of Shell and Spatial Structures, H. Kunieda, Nagoya, 314-315.

Papadrakakis M., (1981). "A Method for the Automatic Evaluation of Dynamic Relaxation Parameters.” Computer Methods in Applied Mechanics and Engineering, 25, 35-48.

Pedretti M., (1998). <http://www.ppeng.ch/base_expo.htm> (July. 15, 2001)

Pellegrino S. (1992). “A Class of Tensegrity Domes.” Int. J. Space Structures, 7(2), 127-142.

Pellegrino S., and Calladine C. R., (1986). "Matrix Analysis of Statically and Kinematically Indeterminate Frameworks.” Int. J. Solids Structures, 22(4), 409-428.

Picon A. (1997). L'Art de l'Ingénieur., Ed.du centre Pompidou, Paris, France (in French), 264.

Robbin J. L., (1996)., Engineering A New Architecture, Yale University Press, Princeton, 25-37.

Rossier S., (1993). "Optimization of cable structures using genetic algorithms." travail pratique de diplôme, Swiss Federal Institute of Technology , Switzerland.

Salama M., Umland J., Bruno R., and Garba J., (1993). "Shape Adjustement of Precision Truss Structures: Analytical and Experimental Validation.” Smart Structures and Materials, 2, 240-248.

Shea K., Fest E., and Smith I. F. C., (2002). "Developing Intelligent Civil structures.” Advanced Engineering Informatics, Elsevier, 16(1).

Shea K., and Smith I., (1998). "Intelligent structures:a new direction in structural control.” Artificial Intelligence in Structural Engineering, LNAI 1454, Computer Science, Springer, Heidelberg, 398-410.

Skelton R.E., Pinaud J.P., and Mingori D.L., (2001). "Dynamics of the Shell Class of Tensegrity Structures.” J. of the Franklin Institute, 338, 255-338.

Snelson K., (1965). “Continuous Tension, Discontinuous Compression Structures.” U.S. Patent N³,169,611.

Snelson K., (1998). <http://www.grunch.net/snelson/> (Aug. 15, 2001).

Sultan C., (1999). "Modeling, Design and Control Tensegrity Structures with Applications.” Purdue University, PhD Thesis.

Swartz W., and Sechen C., (1990). "New Algorithms for the Placement and Routing of Macro Cells.” IEEE Conference on Computer-Aided Design, Santa Clara, USA, 336-339.

Tibert G., (2002).”Deployable Tensegrity Structures for Space Applications”, Doctoral Thesis, Royal Institute of Technology, Department of Mechanics, Stockholm.

Vassart N. , Laporte R. , and Motro R., (2000). "Determination of Mechanism's Order for Kinematically and Statically Indetermined Systems.” Int. J. of Solids and Structures, 37, 3807-3839. 
TABLE 1. Configuration and material characteristics.

TABLE 2. Description of load cases.

TABLE 1. Description of the type, location and magnitude of loads, and the location of displacement measurements. 


\begin{tabular}{lc}
\hline \hline Configuration & Value \\
\hline Number of modules & 3 \\
Number of struts / module & 6 \\
Number of cables / module & 24 \\
Surface & $9 \mathrm{~m}^{2}$ \\
Height & $1.1 \mathrm{~m}$ \\
Mass per area covered & $30 \mathrm{~kg} / \mathrm{m}^{2}$ \\
& Value \\
Struts & Fiberline $\mathrm{TM} P F R$ \\
Material & $70 \mathrm{kN} / \mathrm{mm}^{2}$ \\
Young's modulus & $1800 \mathrm{~kg} / \mathrm{m}^{3}$ \\
Density & $1296 \mathrm{~mm}$ \\
Length & $703 \mathrm{~mm} \mathrm{~m}^{2}$ \\
Cross section & $230.0 \mathrm{~N} / \mathrm{mm}^{2}$ \\
Allowable stress & $20,000 \mathrm{~N}$ \\
Critical buckling force & $\mathrm{Value}$ \\
Cables & Stainless steel \\
\hline Material & $110 \mathrm{kN} / \mathrm{mm}^{2}$ \\
Effective Young's modulus & $0.13 \mathrm{~kg} / \mathrm{m}$ \\
Density & $620 \mathrm{~mm}$ \\
Lengths (3 different) & $1003 \mathrm{~mm}$ \\
& $1516 \mathrm{~mm}$ \\
Cross section & $11.86 \mathrm{~mm} 2$ \\
\hline \hline
\end{tabular}

\begin{tabular}{lcc}
\hline Load Case & Joint & $\begin{array}{c}\text { Load } \\
(\mathrm{N})\end{array}$ \\
\hline 1 - edge & 52 & -860 \\
2- edge & 52 & -623 \\
3 - top/center & 12 & -860 \\
4 - top/center & 12 & -623 \\
\hline \hline
\end{tabular}




\begin{tabular}{lcccc}
\hline \hline Type & $\begin{array}{c}\text { Joint } \\
\text { number }\end{array}$ & $\begin{array}{c}\text { Maximum } \\
\text { load } \\
\text { applied } \\
(\mathrm{N})\end{array}$ & $\begin{array}{c}\text { Load } \\
\text { Steps } \\
(\mathrm{N})\end{array}$ & $\begin{array}{c}\text { Measured } \\
\text { joints } \\
(\mathrm{mm})\end{array}$ \\
\hline 1-Symmetric & 6,52, & 860 & 152 & $7,54,63$, \\
& 62,5, & & and & $6,52,62$, \\
& 48,61 & & 236 & $3,50,59$ \\
2-Asymmetric & 6,52, & & 152 & $7,54,63$, \\
& 62,5, & 1923 & and & $6,52,62$, \\
3-Central joint & 48,61 & & 236 & $3,50,59$ \\
Module 1 & 7 & & & \\
Module 2 & 54 & 860 & and & $7,54,63$, \\
Module 3 & 63 & & 236 & $3,50,62$, \\
4-Adjusting & - & - & - & $7,54,63$, \\
strut lengths & & & & $6,52,62$, \\
& & & & $5,48,61$ \\
\hline \hline
\end{tabular}


FIG. 1. Plan view of three modules. Note that between modules, compression elements do not touch.

FIG. 2. Elevation view showing the three concrete supports.

FIG.3. Description of movements for a telescopic strut.

FIG. 4. Cross sectional view of a cable connection.

FIG. 5. Cross section of a module-connection joint.

FIG. 6. Shape adjustment loop.

FIG. 7 Solution space size. The logarithmic vertical axis indicates that there is an exponential relationship between the number of possible solutions and the number of adjustable struts.

FIG. 8. Plan view: sensor and load location.

FIG. 9. Elevation view: sensor and load location.

FIG. 10. Schema of slope maintenance: (a) 2D view of possible slopes and (b) 3D view of different slopes.

FIG. 11. Testing procedure.

FIG. 12. Correlation between the analytic model and measurements: (a) Displacements of select joints due to a $981 \mathrm{~N}$ load on Joint 7; (b) Displacement of the loaded joint (Joint 7), module 2; (c) Maximum displacement of an unloaded joint (Joint 3), module 3; (d) Displacement of another unloaded joint of module 2 (Joint 52); (e) Displacement of the central joint of the module 1 (Joint 63).

FIG. 13. Behavior under asymmetric loading: (a) Displacements of joints due to a 860N on Joint 62; (b) Displacement of loaded joint 62, module 1; (c) Maximum displacement of all joints is at Joint 59, module 2; (d) Displacement of a symmetric joint (Joint 52), module 2; (e)Displacement of a central joint (Joint 7), module 2.

FIG. 14. Behavior under symmetric loading: (a) Displacements of select joints due to a $860 \mathrm{~N}$ on joints 6, 52 and 62; (b) Displacement of loaded joint (Joint 6); (c) Displacement of the loaded joint (Joint 62); (d) Displacement of the central joint of the module 3 (Joint 54); (e) Displacement of a lower level joint (Joint 3), module 3.

FIG. 15. The inaccuracy of elastic superposition for predicting experimental behavior: (a) Displacements magnitude and direction of different joints due to a 860N on joints 5, 48 and 61; (b) Displacement of Joint 59, superposition loading on joint 5, 48 and 61; (c) Displacement of Joint 3, superposition loading on joint 5, 48 and 61.

FIG. 16. Response error versus number of adjusted struts comparing tests with two prediction methods.

FIG. 17. Response improvement with 5 adjustable struts/per module.

FIG. 18. Response improvement with 2 adjustable struts/per module. 

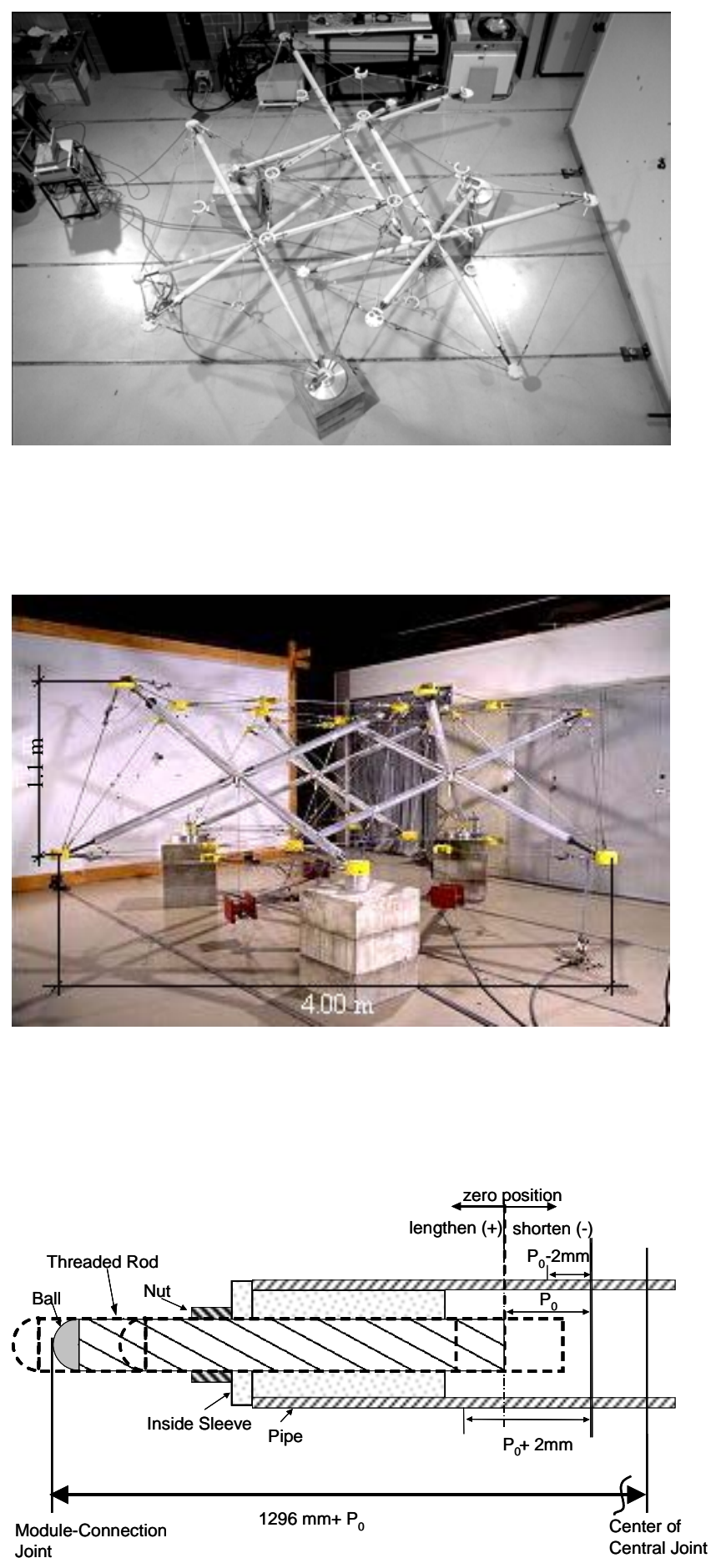

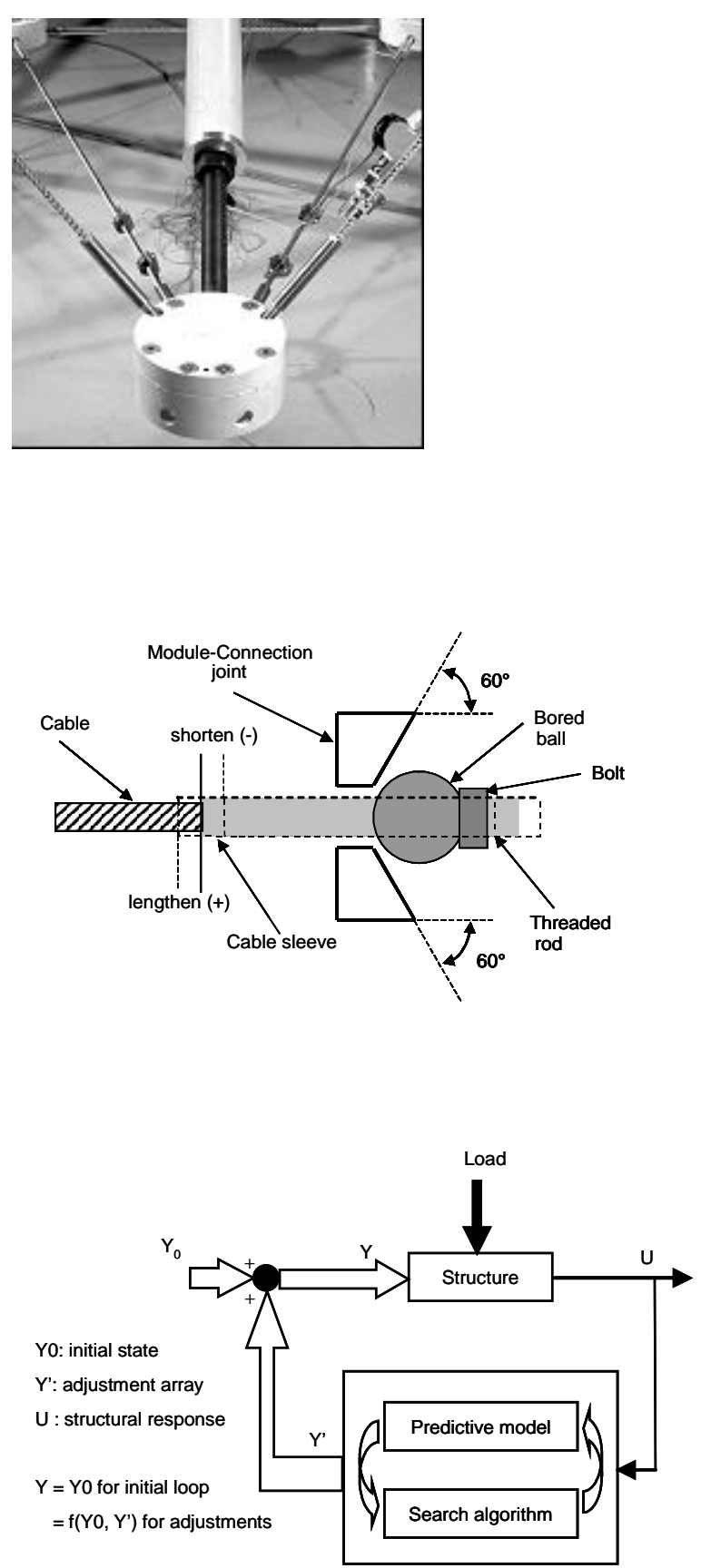


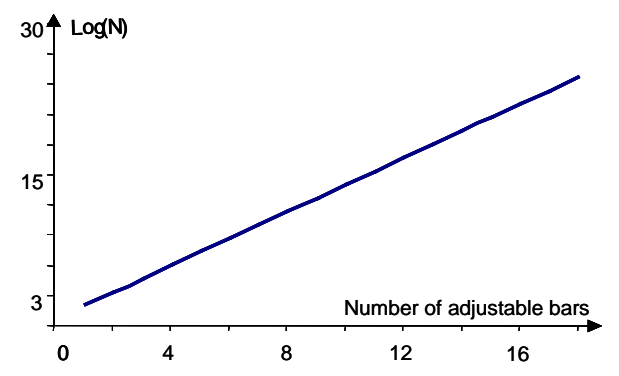



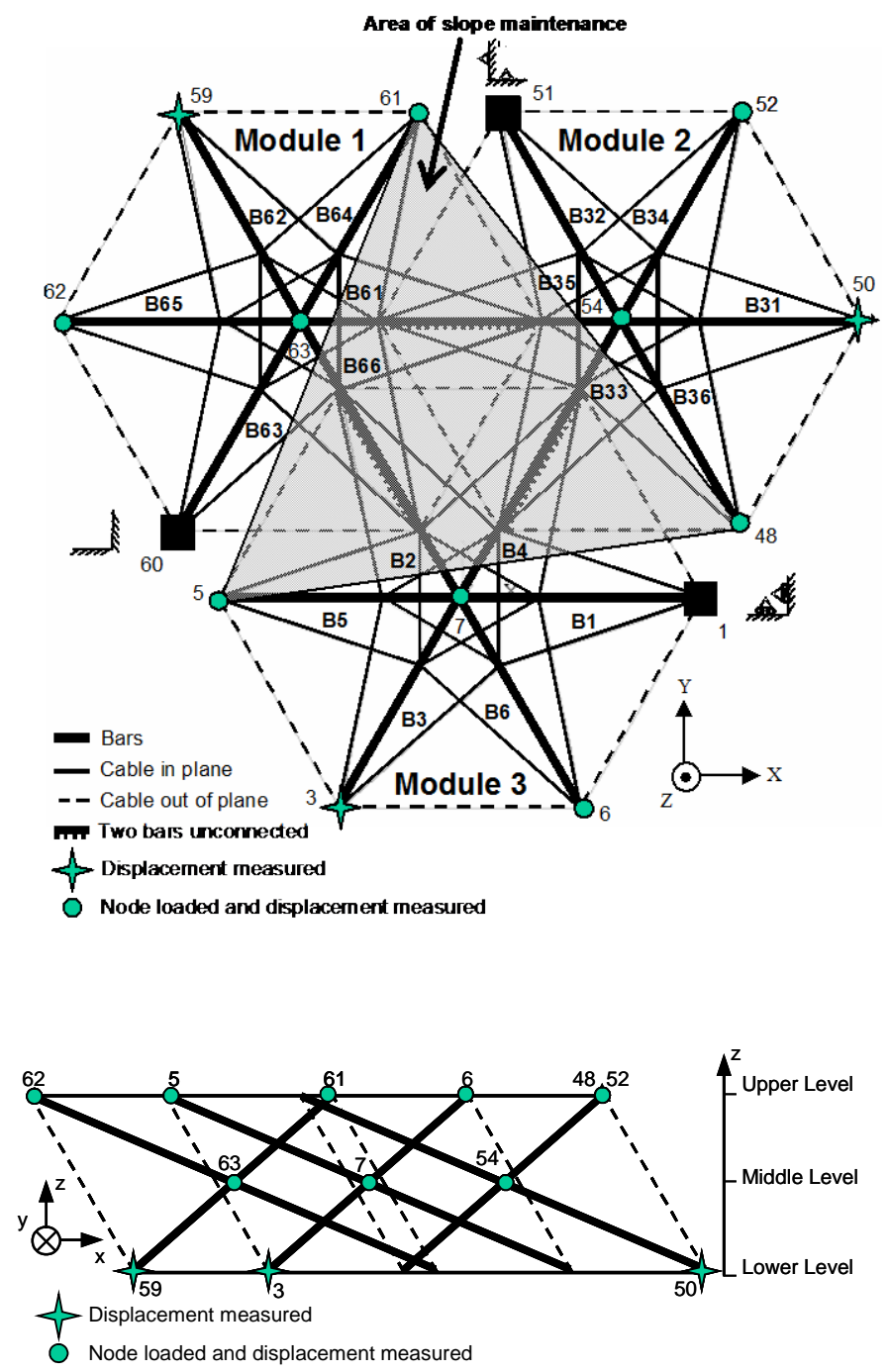


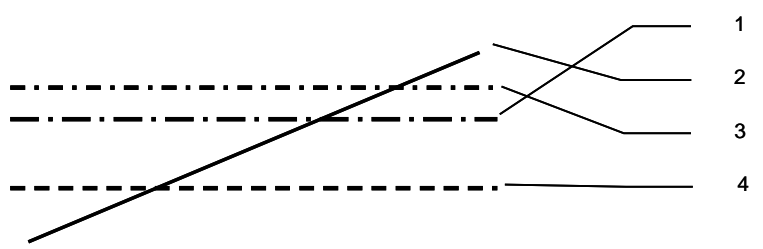

a)

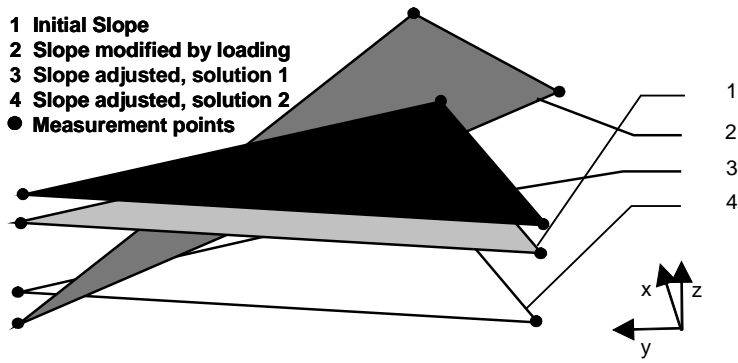

b) 


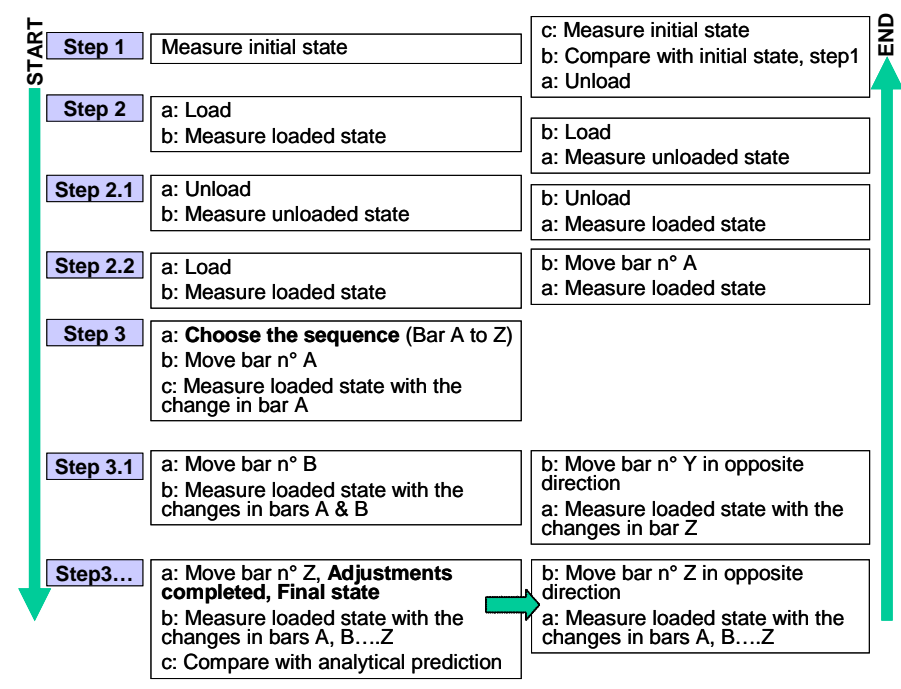

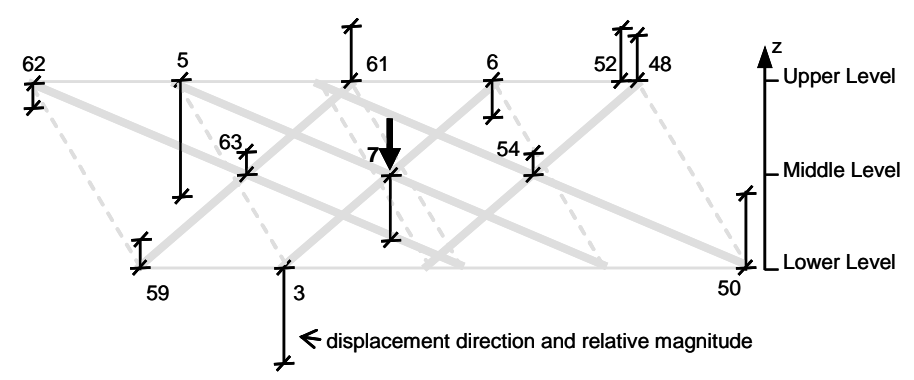

(a)

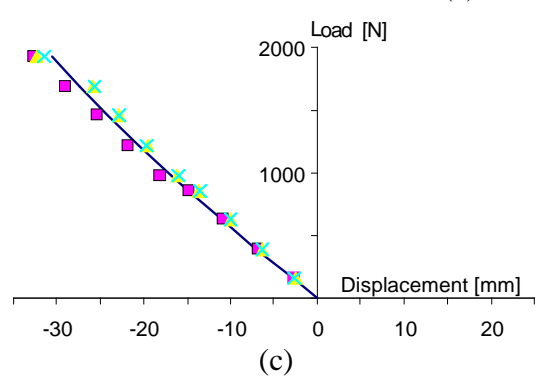

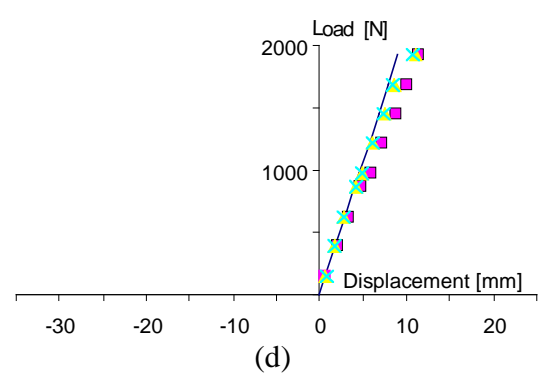

(d)

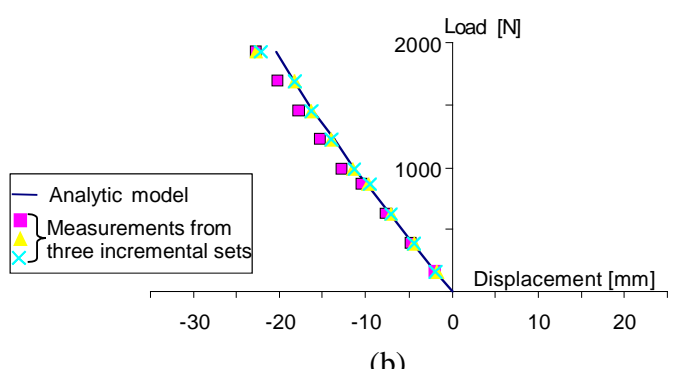

(b)

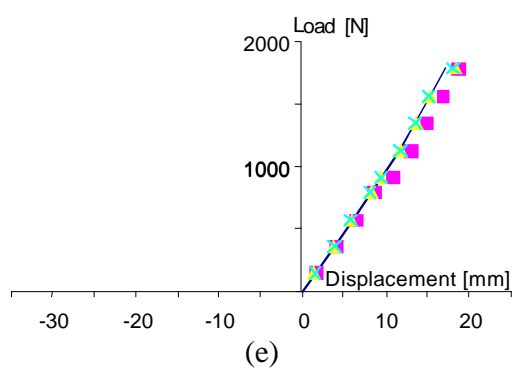




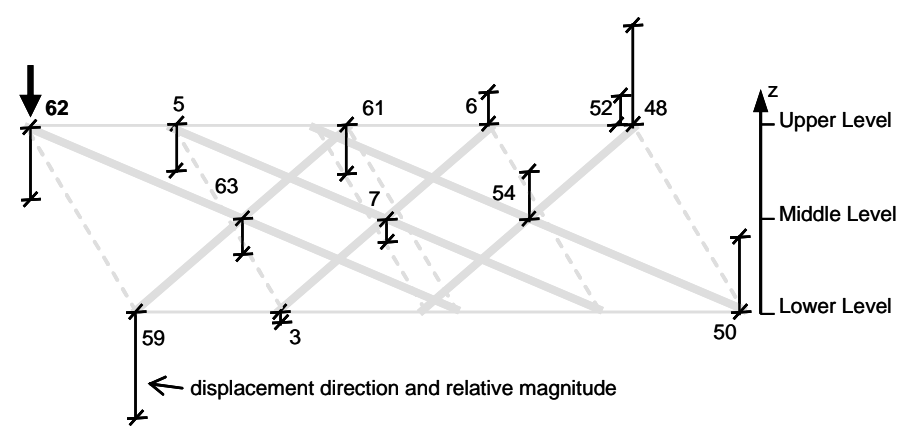

(a) (c)

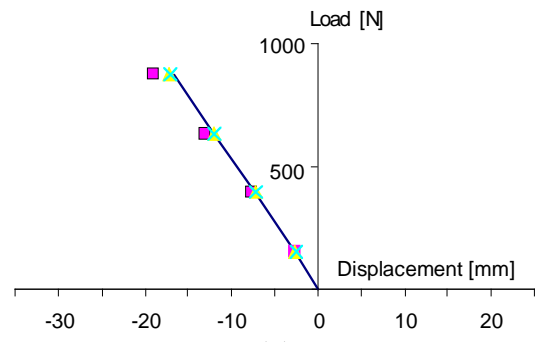

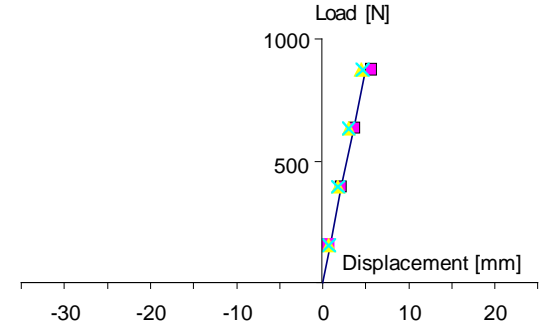

(d)

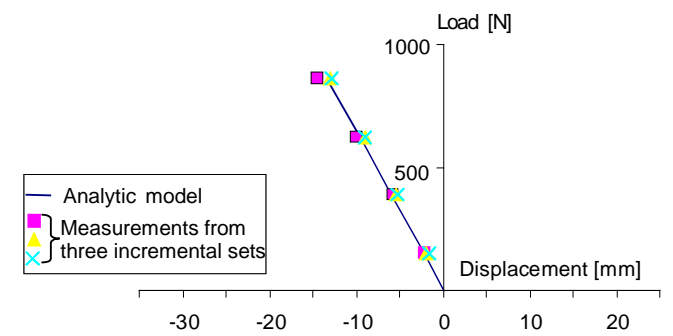

(b)

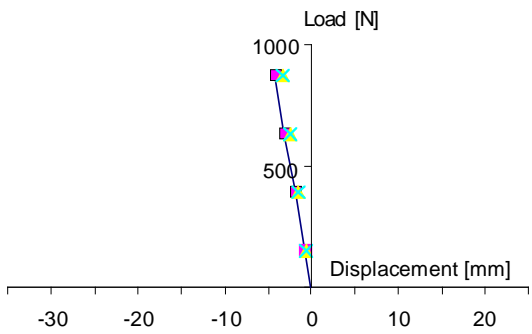

(e)
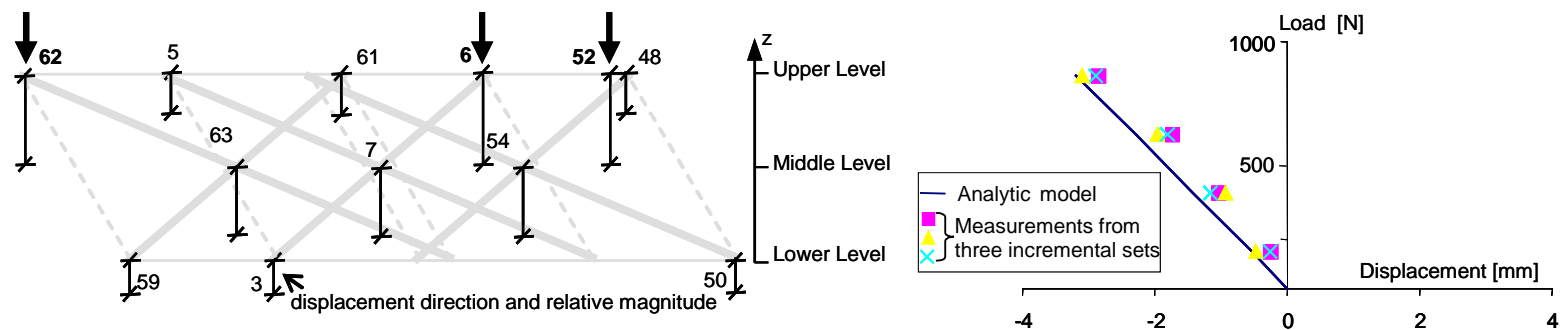

(a)

(b)

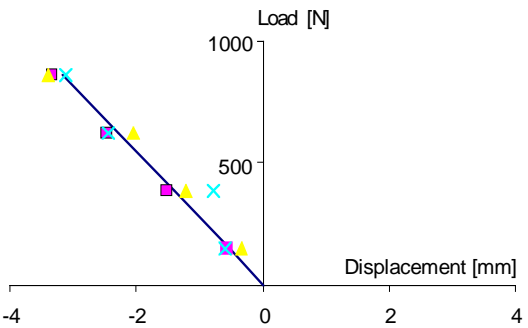

(c)

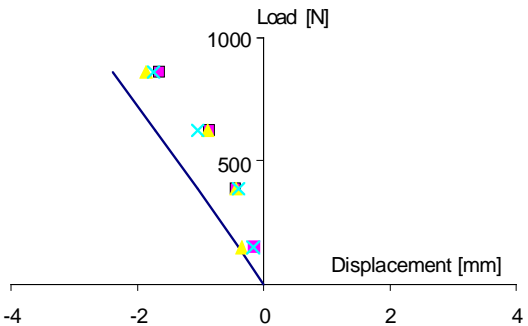

(d)

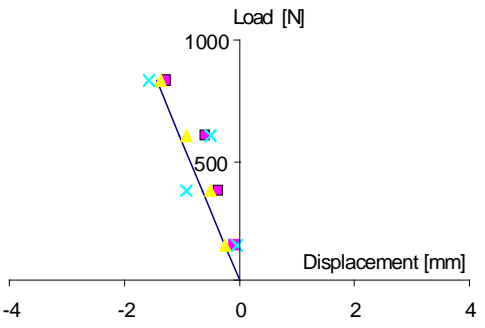

(e) 


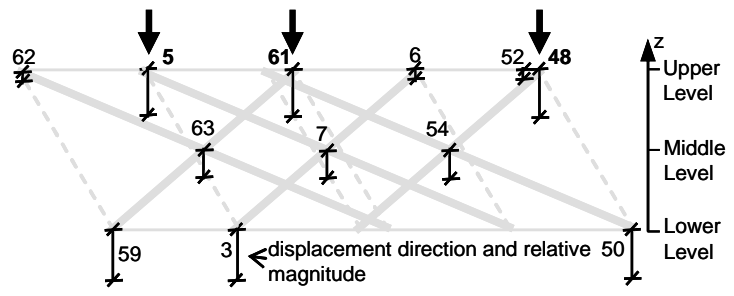

(a)

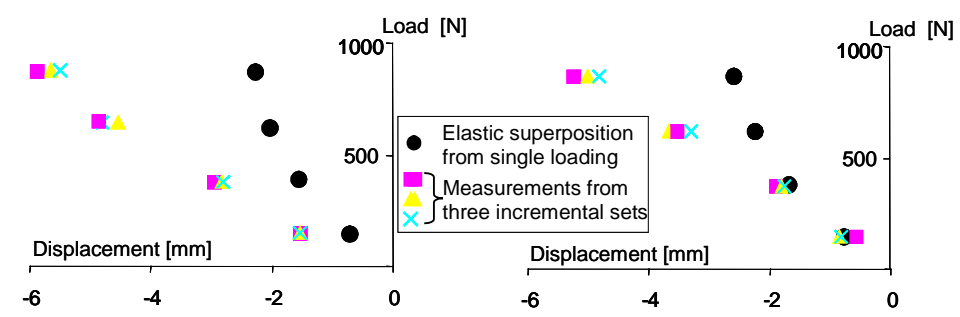

(b) (c) 

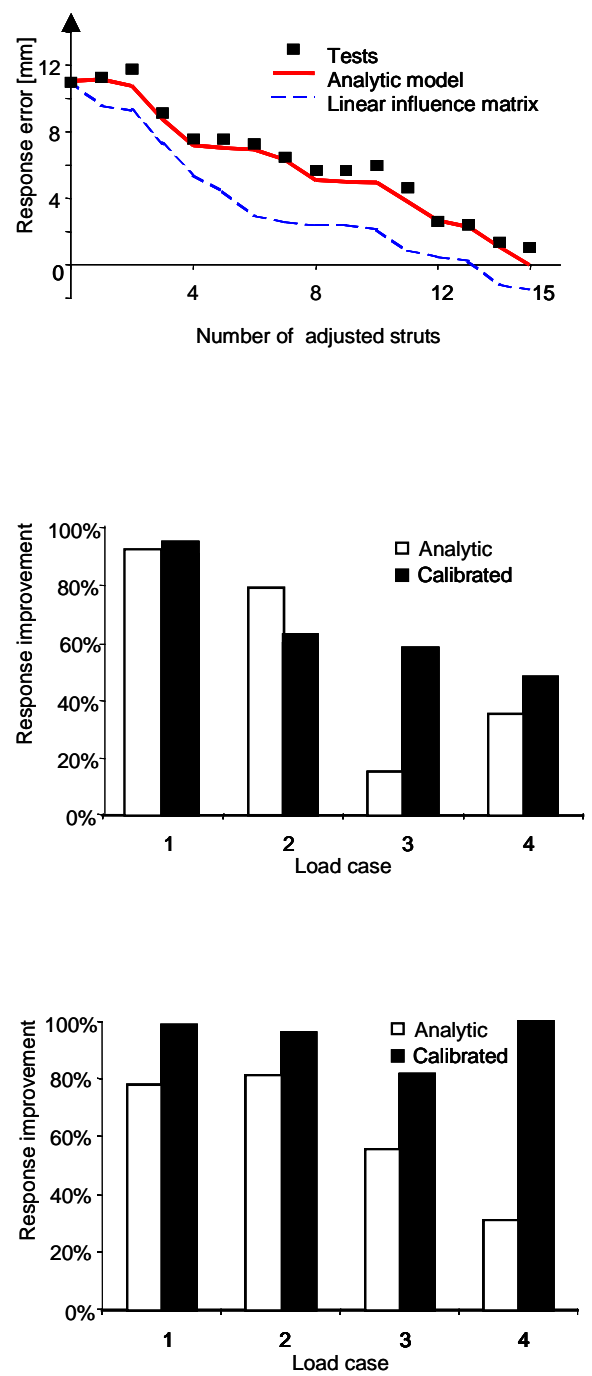